\title{
Aldosterone in Gynecology and Its Involvement on the Risk of Hypertension in Pregnancy
}

\author{
Chiara Sabbadin ${ }^{1}$, Alessandra Andrisani ${ }^{2}$, Guido Ambrosini ${ }^{2}$, Luciana Bordin ${ }^{3}$, \\ Gabriella Donà ${ }^{3}$, Jacopo Manso ${ }^{1}$, Filippo Ceccato ${ }^{1}$, Carla Scaroni ${ }^{1}$ and Decio Armanini ${ }^{1 *}$ \\ ${ }^{1}$ Department of Medicine-Endocrinology, University of Padova, Padua, Italy, ${ }^{2}$ Department of Women's and Children's Health, \\ University of Padova, Padua, Italy, ${ }^{3}$ Department of Molecular Medicine-Biological Chemistry, University of Padova, Padua, \\ Italy
}

Aldosterone is the main mineralocorticoid hormone, responsible of the regulation of fluid and electrolyte balance and blood pressure. It acts also as a pro-inflammatory factor responsible of an increased cardiovascular risk, independent from blood pressure values. After the discovery of mineralocorticoid receptor (MR) in mononuclear leukocytes, further studies supported its role in inflammatory and even autoimmune mechanisms underlying several diseases. In particular, recent studies reported a possible involvement of aldosterone in some gynecological conditions and diseases, characterized by

OPEN ACCESS

Edited by:

GianLuca Colussi,

University of Udine, Italy

Reviewed by:

Francesco Fallo,

University of Padova, Italy

Cristián A. Amador,

Universidad Autónoma de Chile, Chile

*Correspondence:

Decio Armanin

decio.armanini@unipd.it

Specialty section:

This article was submitted to

Translational Endocrinology,

a section of the journal

Frontiers in Endocrinology

Received: 20 April 2019

Accepted: 06 August 2019

Published: 23 August 2019

Citation:

Sabbadin C, Andrisani A Ambrosini G, Bordin L, Donà G, Manso J, Ceccato F, Scaroni $C$ and

Armanini D (2019) Aldosterone in

Gynecology and Its Involvement on the Risk of Hypertension in Pregnancy.

Front. Endocrinol. 10:575.

doi: 10.3389/fendo.2019.00575 inflammation, hypertension and increased cardio-metabolic risk, such as use of hormonal contraceptives, preeclampsia, polycystic ovary syndrome, uterine fibroids, and endometriosis. The aim of this mini-review is to report the possible involvement of aldosterone in all these gynecological conditions, suggesting different pathogenetic mechanisms and new target treatments of MR blockers for these diseases.

Keywords: aldosterone, polycystyc ovary syndrome, hypertension, preeclampsia, spironolactone, endometriosis

Aldosterone (Aldo) is the main mineralocorticoid hormone, synthesized and secreted by the zona glomerulosa of the adrenal cortex in response to angiotensin II and potassium. It was discovered in 1953, few years later the discovery of deoxycorticosterone (DOC), the first mineralocorticoid synthesized and studied for its role on electrolyte balance (1). In 1949 Selye, describing the adaption syndrome, hypothesized that the effect of mineralocorticoids was opposite to that of glucocorticoids being cortisol an anti-inflammatory and DOC a pro-inflammatory hormone (2). Subsequently the putative inflammatory role of Aldo was forgotten and all the studies were focused on its role in sodium balance and hypertension. Only in the last decades several studies reported its involvement in cardiovascular and metabolic disorders, revaluating the initial hypothesis of Selye on Aldo as a pro-inflammatory hormone.

Inflammation is a complex systemic process involving blood vessels, immune cells and molecular mediators. It is involved not only in response to harmful triggers, such as pathogens, damaged cells or toxic agents, but also in autoimmunity, somatic mutations, and cancer (3). In 1985 we characterized mineralocorticoid receptor (MR) in mononuclear leukocytes (MNL) and demonstrated that Aldo can regulate the intracellular concentration of electrolytes and the volume of MNL (4-7). In 2004 we reported that incubation of MNL with Aldo can induce in vitro the protein expression of some inflammatory markers, as PAI-1 and p22phox (8). This effect was reversed by coincubation with canrenone, the main active metabolite of spironolactone (SP), a MR blocker. Aldo can exert its effects in non-epithelial cells, such as cardiomyocytes, endothelial cells, vascular 
smooth muscle cells (VSMC) and mesangial cells, leading to fibrosis and remodeling in the heart, vasculature, and kidney. In particular, at the level of the vessel Aldo cross talks with angiotensin II and endothelin-1, inducing inflammation and oxidative stress, regulating cell proliferation, and leading to endothelial dysfunction (9). These effects are mediated through genomic and non-genomic pathways in a MR-dependent or independent manner, as demonstrated for example on erythrocytes, where Aldo induced in a dose- and time-dependent manner membrane alterations, almost completely prevented by canrenone or cortisol $(10,11)$. These membrane alterations were observed even in erythrocytes of patients affected by primary aldosteronism (PA) and led to increased autologous IgG binding, responsible for premature erythrocyte removal from circulation.

Further studies demonstrated that PA patients present an increased risk of stroke, myocardial infarction, atrial fibrillation, and even metabolic syndrome compared with appropriately matched controls with essential hypertension (12, 13). In fact, Aldo is also involved in the development of several metabolic alterations, impairing insulin secretion and sensitivity, altering potassium levels, increasing inflammatory cytokines and reducing adiponectin (14). Treatment with MR blockers improves cardiovascular and metabolic outcomes similar to surgical treatment of PA $(14,15)$. In the end of 1990th Pitt and coll. confirmed the importance of MR blockers to prevent cardiovascular complications and cerebrovascular accidents even in patients with normal values of Aldo $(16,17)$ and several studies are evaluating a possible terapeuthic role of MR blockers to treat obesity and metabolic disorders (18).

Recently, Herrada et al. reported that T-cells are also regulated by Aldo, promoting CD4 T-cell activation and T-helper 17 cell formation (19). T-helper 17 are a subset of CD4 T-cells producing interleukin 17 , that is involved in many autoimmune diseases, such as Hashimoto's thyroiditis (20). Recently, we reported that PA patients have an increased prevalence of Hashimoto's thyroiditis (21) and an increased titer of autoantibodies against the angiotensin II type 1 receptor (AT1-AA), that could have some pathogenetic role in PA and related complications (22). From all these studies the important role of Aldo in the genesis of inflammation and related diseases seems mostly mediated by the presence of MR in MNL and macrophages.

The aim of this mini-review is to report the involvement of Aldo in the hypertension induced by pregnancy and in other gynecological diseases frequently associated with hypertension and inflammatory dysregulation.

\section{ALDOSTERONE IN PREGNANCY AND PREECLAMPSIA}

During physiological pregnancy, Aldo levels are increased to induce plasma volume expansion, crucial for maintaining circulating blood volume, blood pressure, and uteroplacental perfusion. These mechanisms are supported already from the first trimester by increased renin production by kidney in response to several cardiovascular adaptations and by increased angiotensinogen secretion by the liver driven by placental production of estrogens (23). This activation of the renin angiotensin Aldo system (RAAS) during pregnancy increases 3 - to 7-fold compared to initial values. Further studies have also demonstrated an increased Aldo-to-renin ratio (ARR) in physiological pregnancy, suggesting additional factors which stimulate Aldo production and one of them could be the vascular endothelial growth factor (VEGF) (24). Aldo levels remain high throughout pregnancy, suggesting a possible role in the regulation of placental and fetal development (25). Despite increased Aldo levels, healthy pregnant women do not usually present hypertension through several compensatory mechanisms. The MR-antagonist actions of progesterone and the increased glomerular filtration rate induce natriuresis despite the sodium retaining properties of Aldo. Moreover, in physiological pregnancy angiotensin II shows a reduced pressor effect.

In gestational hypertension and in preeclampsia (PE), differently from physiological pregnancy, decreased RAAS activity and increased sensitivity to angiotensin II effects are found, resulting in decreased plasma volume expansion, vascular constriction, reduction of cardiac output and decreased renal and placental blood flow (23). In particular, PE is the most severe form of hypertension in pregnancy. It is characterized by the new onset of hypertension and proteinuria usually after 20 weeks of pregnancy. The pathogenesis is still debated and remains unknown. Genetic, epigenetic, inflammatory, and autoimmune causes have been involved in the pathogenesis. Several predisposing factors have also been reported, such as chronic arterial hypertension, chronic kidney disease, obesity, diabetes, polycystic ovary syndrome (PCOS), endometriosis, familial or personal history of PE (26). Besides placental abnormalities, many studies suggest a possible role of the RAAS in the pathogenesis of PE. As previously reported, both Aldo and progesterone are high during pregnancy, but the number of MR in MNL has been found normal in physiological pregnancy and low in PE (27), as reported in patients with PA (28). More interestingly the effector mechanism of Aldo, measured through the potential difference between rectal and oral mucosa, was normal in normal pregnancy and high in $\mathrm{PE}$, as previously reported in PA (29). We hypothesized that some factor produced by the placenta blunts the effect of increased Aldo in normal pregnancy, but it lacks in $\mathrm{PE}$, leading to the classical effects of Aldo excess, even though Aldo levels are reduced. Other possible explanations could be a reduced antagonist effect of progesterone at the level of MR in PE (30) or an impaired VEGF-mediated stimulation of aldosterone synthase (24). From these considerations a possible therapeutic role of MR blockers in PE has been hypothesized (31). Unfortunately, SP has a potent antiandrogen effect and both canrenone and potassium canrenoate have a weaker antiandrogen action, which could alter male fetus morphogenesis $(32,33)$. Eplerenone could be the preferred MR blocker in pregnancy, showing no-antiandrogen effect (31). Indeed, isolated reports show its efficacy to manage blood pressure in PA during pregnancy (34). However, women with PA usually show an improvement in blood pressure values and hypokaliemia during pregnancy, probably because of the antagonist effect of increased progesterone levels at the level of MR (35). 
Another interesting hypothesis of the pathogenesis of $\mathrm{PE}$ was postulated by Wallukat et al. (36), who firstly described in preeclamptic women the presence of AT1-AA, which were able to induce a preeclamptic state when injected in pregnant mice (37). The subsequent discovery of increased titers of AT1-AA even in PA patients (22) leads to a possible common underlying etiopathogenesis of PE and PA. The possible pathogenetic role of AT1-AA is complex and could be related to the activation of AT1 receptor, leading to increased vascular resistance and endothelial damage, due to increased production of many pro-oxidative and pro-inflammatory factors. The interplay between placental abnormalities, oxidative stress and endothelial dysfunction are key events in the development of PE, but more studies are needed to better understand the etiology of this complex disease (38).

\section{ALDOSTERONE IN OTHER GYNECOLOGICAL CONDITIONS}

Recent studies have reported a possible involvement of Aldo in other gynecological diseases, sometimes associated with inflammation, hypertension and actual or future cardiometabolic risk, such as the use of hormonal contraceptives, PCOS, uterine fibroids and endometriosis.

\section{ALDOSTERONE AND HORMONAL CONTRACEPTIVES}

Hypertension was a frequent complication of the hormonal contraceptives with high ethinilestradiol content and it is actually a rare side effect of contraceptives with lower amounts of ethinilestradiol (20-30 $\mu \mathrm{g})$, but still reported in some cases, stressing out the implication of a genetic predisposition. It is well known that estrogens increase both cortisol and Aldo. Cortisol increases in response to the increased level of cortisol binding globulins in order to maintain its free quote in the normal range. The mechanism involved in the increase of Aldo during contraceptives is not completely clarified. The most likely hypothesis is that estrogens increase the synthesis of angiotensinogen, activating all the RAAS and inducing the onset of sodium and water retention, metabolic alterations and sometimes hypertension (39). For all these reasons, it is recommended to avoid the use of hormonal contraceptives in patients with hypertension and metabolic alterations.

\section{ALDOSTERONE AND PCOS}

PCOS is a very common disease affecting about $10 \%$ of women in the reproductive age. It is characterized by oligo-anovulation and biochemical/clinical signs of hyperandrogenism. Several phenotypes have been described, but a common feature is insulin resistance, interesting about $70 \%$ of patients even normoweight and representing one of the main pathogenetic factors of the syndrome (40). Beyond the reproductive disorders, even at an early stage PCOS patients can present a clustering of cardiovascular risk factors, such as insulin resistance, obesity, diabetes, dyslipidemia, hypertension, endothelial dysfunction, and low-grade chronic inflammation (41). Previous studies found that Aldo and ARR, though normal and not consistent with PA, are higher in PCOS women compared with age- and body mass index-matched healthy controls and correlate with blood pressure values and some metabolic and cardiovascular markers $(42,43)$. As previously reported, Aldo plays a key role as pro-inflammatory hormone and cardiovascular and metabolic risk factor $(12,13)$ and could play a certain role in the development of many Aldo-related disorders even in PCOS, such as hypertension, PE, metabolic alterations, Hashimoto's thyroiditis and cardiovascular diseases. Moreover, we recently reported that PCOS erythrocyte membranes showed an increased oxidized level and enhanced sensitivity to oxidative injuries $(41,44)$, as reported in PA patients' erythrocytes, confirming a systemic inflammatory status $(10,11)$.

The treatment of PCOS should take into consideration not only the acute manifestations of the syndrome, like menstrual disorders and hirsutism, but also the related long-term cardiac and metabolic complications, that could be worsened in pregnancy and after menopause. Hormonal contraceptives are first-line management for the menstrual abnormalities and hyperandrogenic disorders of PCOS patients (45). However, their role on insulin resistance is controversial and considering their activation of RAAS, it could be hypothesized a further increase of Aldo levels in PCOS patients, that could worsen some cardio-metabolic aspects of the disease. Therefore, their use should be carefully evaluated for every single patients, after considering all the pre-existing metabolic and cardiovascular risk factors. We have reported that SP is useful both in controlling the clinical signs of hyperandrogenism and in preventing the future cardiovascular risk (46). Its main side effects are hypotension and hyperkalemia, due to MR block, and frequent menstrual abnormalities, due to a complex effect on estradiol and progesterone actions (47). Because of the potential risk to the male fetus due to its antiandrogen effect, women must be informed to take precautions to avoid pregnancy during SP treatment. The association of SP with hormonal contraceptives is useful for many reasons: it enhances the antiandrogen effects, it prevents unwanted pregnancies and menstrual abnormalities linked to SP and it reduces the side effects related to the activation of RAAS induced by some contraceptives, such as sodium and water retention, but even hypertension and cardio-metabolic alterations. When hormonal contraceptives are controindicated, the addition of low dose progestins from the 14th day of the cycle can block the bleeding induced by SP $(46,47)$ and the use of licorice can prevent hypotension and hyperkalemia, blocking the $11 \beta$-hydroxysteroid dehydrogenase type 2 , and can enhance the antiandrogen effect, blocking the 17-hydroxysteroid dehydrogenase and 17-20 lyase in adrenals and ovaries (48-51).

\section{ALDOSTERONE AND UTERINE FIBROIDS}

Uterine fibroids (UF) are benign tumors of the muscle layer of uterus, affecting about $70 \%$ of women by age 50 years but being symptomatic only in half of the cases. UF are frequently associated with familial predisposition, obesity, early 
menarche, low parity, and are influenced by the effect of several hormones. Progesterone stimulates cellular proliferation and UF growth (52) and the treatment with progesterone receptor (PR) antagonist mifepristone (RU486) or with the selective PR modulator ulipristal acetate is effective in reducing UF size and collagen deposits and in controlling uterine bleeding (53). Local and general inflammation have been associated with UF development and T-helper 2 bias in the uterine environment has been hypothesized to contribute to UF formation, independent of the sex steroid status (54). A recent study has evidenced an involvement of Aldo in UF, showing a significant stimulatory effect on leiomyoma cell proliferation, which was blocked by the pre-incubation with SP or eplerenone (55). These in vitro studies suggest a possible use of MR blockers in the treatment of UF; however, only eplerenone could be considered, because of the frequent metrorrhagia associated with SP (47). Recently a higher risk of hypertension has been reported in patients with UF (56). However, a previous study evidenced that hysterectomy did not influence the risk of hypertension and on the contrary it was associated with long-term increase of cardiovascular risk (57). These studies are consistent with possible extra-uterine mechanisms involved in hypertension in UF. It is unlikely an involvement of progesterone because of its MR antagonism, while Aldo could be the pathogenetic factor common to hypertension and UF and could explain the association of UF with other Aldo-related disorders, such as insulin resistance, obesity and PCOS (58).

\section{ALDOSTERONE AND ENDOMETRIOSIS}

Endometriosis is a complex syndrome characterized by an estrogen-dependent chronic inflammatory process, caused by the presence of endometrial-like tissue out the uterine cavity. It is the most common cause of chronic pelvic pain in women and it is associated with infertility. Several studies have underlined an important role of inflammation in the pathogenesis of the disease (59). Chronic inflammation is a risk factor for several diseases, as atherosclerosis, diabetes, hypertension, hypothyroidism, autoimmune diseases and cancer and it is reported that endometriosis is more frequently associated with all these disorders (60). Hormonal abnormalities or chronic systemic inflammation characterizing endometriosis may result in a higher risk of hypercholesterolemia and hypertension. Conversely, hypercholesterolemia and chronic systemic inflammation resulting from hypertension may increase the risk

\section{REFERENCES}

1. Sabbadin C, Calò LA, Armanini D. The story of spironolactones from 1957 to now: from sodium balance to inflammation. G Ital Nefrol. (2016) 33(Suppl. 66):12.

2. Selye H. Anticortisol action of aldosterone. Science. (1955) 121:368-9. doi: 10.1126/science.121.3141.368

3. Herrada AA, Campino C, Amador CA, Michea LF, Fardella CE, Kalergis AM. Aldosterone as a modulator of immunity: implications in the organ damage. $J$ Hypertens. (2011) 29:1684-92. doi: 10.1097/HJH.0b013e32834a4c75 of endometriosis (61). A recent study using metabolomics has shown that Aldo is higher in infertile patients with endometriosis (62). As previously demonstrated (8), Aldo could enhance the systemic and local inflammation underlying endometriosis, through the activation of MR present in peritoneal and tissue inflammatory cells, in particular macrophages and MNL. Moreover, Aldo could increase oxidative stress through MRdependent and -independent pathways in VMSC, that promote proliferation of fibroblasts in the perivascular space (63). A recent study in an animal model of continuous ambulatory peritoneal dialysis demonstrated that SP was effective at decreasing intraperitoneal fibrosis and inflammation (64). Considering the substantial experience with the safe chronic use of SP in women with androgen excess disorders as PCOS, investigators should evaluate the therapeutic potential of SP or other MR blockers in endometriosis. In our experience, SP is also very useful in the dysmenorrhea probably for its powerful anti-inflammatory effect.

\section{CONCLUSIONS}

In the recent years Aldo has been considered an important pro-inflammatory hormone, involved in many conditions characterized by chronic inflammation, as cardio-metabolic and some gynecological disorders. Inflammation starts in the circulation activating MNL and macrophages, that migrate in inflammatory tissues, presenting their MR that can be activated by Aldo, enhancing the inflammation $(5-7,63)$. The finding of a reversal of inflammatory properties of Aldo by coincubation or treatment with MR blockers is a further confirmation of the important role of Aldo. In the recent years the normal range of Aldo has been revisited $(3,4)$, stressing the importance of its ratio with renin, that could represent a sign of enhanced Aldo effector mechanism when it is increased (43). Further studies are needed to evaluate the therapeutic potential of MR blockers even as antiinflammatory drugs, especially in some gynecological diseases characterized by a pro-inflammatory state and associated with hypertension and an increased cardio-metabolic risk, that could be related to Aldo dysregulation.

\section{AUTHOR CONTRIBUTIONS}

All authors listed have made a substantial, direct and intellectual contribution to the work, and approved it for publication.
4. Armanini D, Strasser T, Weber PC. Characterization of aldosterone binding sites in circulating human mononuclear leukocytes. Am J Physiol. (1985) 248:E388-90. doi: 10.1152/ajpendo.1985.248.3. E388

5. Armanini D, Wehling M, Weber PC. Mineralocorticoid effector mechanism in human mononuclear leukocytes. J Steroid Biochem. (1987) 27:967-70. doi: 10.1016/0022-4731(87)90175-0

6. Wehling M, Armanini D, Strasser T, Weber PC. Effect of aldosterone on sodium and potassium concentrations in human mononuclear leukocytes. Am J Physiol. (1987) 252:E505-8. doi: 10.1152/ajpendo.1987.252.4.E505 
7. Wehling M, Kuhls S, Armanini D. Volume regulation of human lymphocytes by aldosterone in isotonic media. Am J Physiol. (1989) 257:E170-4. doi: 10.1152/ajpendo.1989.257.2.E170

8. Calò LA, Zaghetto F, Pagnin E, Davis PA, De Mozzi P, Sartorato P, et al. Effect of aldosterone and glycyrrhetinic acid on the protein expression of PAI-1 and p22(phox) in human mononuclear leukocytes. J Clin Endocrinol Metab. (2004) 89:1973-6. doi: 10.1210/jc.2003-031545

9. Briet M, Schiffrin EL. Vascular actions of aldosterone. J Vasc Res. (2013) 50:89-99. doi: 10.1159/000345243

10. Bordin L, Donà G, Sabbadin C, Ragazzi E, Andrisani A, Ambrosini G, et al. Human red blood cells alterations in primary aldosteronism. J Clin Endocrinol Metab. (2013) 98:249-501. doi: 10.1210/jc.2012-3571

11. Bordin L, Saccardi C, Donà G, Sabbadin C, Andrisani A, Ambrosini G, et al. Mineralocorticoid receptor is involved in the aldosterone pathway in human red blood cells. Am J Transl Res. (2016) 8:314-28.

12. Milliez P, Girerd X, Plouin PF, Blacher J, Safar ME, Mourad JJ. Evidence for an increased rate of cardiovascular events in patients with primary aldosteronism. J Am Coll Cardiol. (2005) 45:1243-8. doi: 10.1016/j.jacc.2005.01.015

13. Fallo F, Veglio F, Bertello C, Sonino N, Della Mea P, Ermani M, et al. Prevalence and characteristics of the metabolic syndrome in primary aldosteronism. J Clin Endocrinol Metab. (2006) 91:454-9. doi: 10.1210/jc.20051733

14. Luther JM. Effects of aldosterone on insulin sensitivity and secretion. Steroids. (2014) 91:54-60. doi: 10.1016/j.steroids.2014.08.016

15. Marzano L, Colussi G, Sechi LA, Catena C. Adrenalectomy is comparable with medical treatment for reduction of left ventricular mass in primary aldosteronism: meta-analysis of long-term studies. Am J Hypertens. (2015) 28:312-8. doi: 10.1093/ajh/hpu154

16. Pitt B, Zannad F, Remme WJ, Cody R, Castaigne A, Perez A, et al. The effect of spironolactone on morbidity and mortality in patients with severe heart failure. N Engl J Med. (1999) 341:709-17. doi: 10.1056/NEJM199909023411001

17. Pitt B, Remme W, Zannad F, Neaton J, Martinez F, Roniker B, et al. Eplerenone, a selective aldosterone blocker, in patients with left ventricular dysfunction after myocardial infarction. N Engl J Med. (2003) 348:1309-21. doi: 10.1056/NEJMoa030207

18. Marzolla V, Armani A, Feraco A, De Martino MU, Fabbri A, Rosano $\mathrm{G}$, et al. Mineralocorticoid receptor in adipocytes and macrophages: a promising target to fight metabolic syndrome. Steroids. (2014) 91:46-53. doi: 10.1016/j.steroids.2014.05.001

19. Herrada AA, Contreras FJ, Marini NP, Amador CA, González PA, Cortés $\mathrm{CM}$, et al. Aldosterone promotes autoimmune damage by enhancing Th17-mediated immunity. J Immunol. (2010) 184:191-202. doi: 10.4049/jimmunol.0802886

20. Shi Y, Wang H, Su Z, Chen J, Xue Y, Wang S, et al. Differentiation imbalance of Th1/ Th17 in peripheral blood mononuclear cells might contribute to pathogenesis of hashimoto's thyroiditis. Scand J Immunol. (2010) 72:250-5. doi: 10.1111/j.1365-3083.2010.02425.x

21. Sabbadin C, Mian C, Nacamulli D, Donà G, Presotto F, Betterle C, et al. Association of primary aldosteronism with chronic thyroiditis. Endocrine. (2017) 55:303-6. doi: 10.1007/s12020-016-0880-2

22. Sabbadin C, Ceccato F, Ragazzi E, Boscaro M, Betterle C, Armanini D. Evaluation of angiotensin II type-1 receptor antibodies in primary aldosteronism and further considerations about their possible pathogenetic role. J Clin Hypertens. (2018) 20:1313-8. doi: 10.1111/jch. 13351

23. Swiatkowska-Stodulska R, Kmieć P, Stefanska K, Sworczak K. Reninangiotensin-aldosterone system in the pathogenesis of pregnancyinduced hypertension. Exp Clin Endocrinol Diabetes. (2018) 126:362-6. doi: $10.1055 / \mathrm{s}-0044-102008$

24. Gennari-Moser C, Khankin EV, Escher G, Burkhard F, Frey BM, Karumanchi $\mathrm{SA}$, et al. Vascular endothelial growth factor-A and aldosterone: Relevance to normal pregnancy and preeclampsia. Hypertension. (2013) 61:1111-7. doi: 10.1161/HYPERTENSIONAHA.111.00575

25. Gennari-Moser C, Khankin EV, Schuller S, Escher G, Frey BM, Portmann $\mathrm{CB}$, et al. Regulation of placental growth by aldosterone and cortisol. Endocrinology. (2011) 152:263-71. doi: 10.1210/en.2010-0525
26. Armanini D, Andrisani A, Ambrosini G, Donà G, Bordin L, Sabbadin C. Hypertension in pregnancy: Role of body mass index, insulin resistance, aldosterone, and calcium homeostasis. J Clin Hypertens. (2019) 21:624-6. doi: $10.1111 /$ jch. 13538

27. Armanini D, Zennaro CM, Martella L, Scali M, Pratesi C, Grella PV, et al. Mineralocorticoid effector mechanism in preeclampsia. J Clin Endocrinol Metab. (1992) 74:946-9.

28. ArmaninI D, Witzgall H, Wehling M, Kuhnle U, Weber PC. Aldosterone receptors in different types of primary hyperaldosteronism. J Clin Endocrinol Metab. (1987) 65:101-4. doi: 10.1210/jcem-65-1-101

29. Beevers DG, Morton JJ, Tree M, Young J. Rectal potential difference in the diagnosis of aldosterone excess. Gut. (1975) 16:36-41. doi: 10.1136/gut.16.1.36

30. Armanini D, Calò LA. Aldosterone, inflammation, and preeclampsia. Hypertension. (2005) 45:e10. doi: 10.1161/01.HYP.0000157170.94539.ee

31. Riester A, Reincke M. Progress in primary aldosteronism: mineralocorticoid receptor antagonists and management of primary aldosteronism in pregnancy. Eur J Endocrinol. (2015) 172:R23-30. doi: 10.1530/EJE-14-0444

32. Armanini D, Karbowiak I, Goi A, Mantero F, Funder JW. In-vivo metabolites of spironolactone and potassium canrenoate: determination of potential antiandrogenic activity by a mouse kidney cytosol receptor assay. Clin Endocrinol. (1985) 23:341-7. doi: 10.1111/j.1365-2265.1985.tb01090.x

33. Armanini D, Sabbadin C, Donà G, Clari G, Bordin L. Aldosterone receptor blockers spironolactone and canrenone: two multivalent drugs. Expert Opin Pharmacother. (2014) 15:909-12. doi: 10.1517/14656566.2014.896901

34. Gunganah $\mathrm{K}$, Carpenter R, Drake WM. Eplerenone use in primary aldosteronism during pregnancy. Clin Case Reports. (2016) 4:81-2. doi: $10.1002 / \mathrm{ccr} 3.355$

35. Armanini D, Sabbadin C, Donà G, Andrisani A, Ambrosini G, Bordin L. Maternal and fetal outcomes in preeclampsia: interrelations between insulin resistance, aldosterone, metabolic syndrome, and polycystic ovary syndrome. J Clin Hypertens. (2015) 17:783-5. doi: 10.1111/jch.12613

36. Wallukat G, Homuth V, Fischer T, Lindschau C, Horstkamp B, Jupner A, et al. Patients with preeclampsia develop agonistic autoantibodies against the angiotensin AT1 receptor. J Clin Invest. (1999) 103:945-52. doi: $10.1172 / J C I 4106$

37. Zhou CC, Zhang Y, Irani RA, Zhang H, Mi T, Popek EJ, et al. Angiotensin receptor agonistic autoantibodies induce pre-eclampsia in pregnant mice. Nat Med. (2008) 14:855-62. doi: 10.1038/nm.1856

38. Sánchez-Aranguren LC, Prada CE, Riaño-Medina CE, Lopez M. Endothelial dysfunction and preeclampsia: role of oxidative stress. Front Physiol. (2014) 5:372. doi: $10.3389 /$ fphys.2014.00372

39. Hassan E, Creatsas G, Mastorakos G, Michalas S. Clinical implications of the ovarian/endometrial renin-angiotensin-aldosterone system. Ann N Y Acad Sci. (2000) 900:107-18. doi: 10.1111/j.1749-6632.2000.tb06221.x

40. Zulian E, Sartorato P, Benedini S, Baro G, Armanini D, Mantero F, et al. Spironolactone in the treatment of polycystic ovary syndrome: effects on clinical features, insulin sensitivity, and lipid profile. J Endocrinol Invest. (2005) 28:49-53. doi: 10.1007/BF03345529

41. Donà G, Sabbadin C, Fiore C, Bragadin M, Giorgino FL, Ragazzi E, et al. Inositol administration reduces oxidative stress in erythrocytes of patients with polycystic ovary syndrome. Eur J Endocrinol. (2012) 166:703-10. doi: 10.1530/EJE-11-0840

42. Cascella T, Palomba S, Tauchmanovà L, Manguso F, Di Biase S, Labella D, et al. Serum aldosterone concentration and cardiovascular risk in women with polycystic ovarian syndrome. J Clin Endocrinol Metab. (2006) 91:4395-400. doi: 10.1210/jc.2006-0399

43. Armanini D, Bordin L, Donà G, Sabbadin C, Bakdounes L, Ragazzi E, et al. Polycystic ovary syndrome: implications of measurement of plasma aldosterone, renin activity and progesterone. Steroids. (2012) 77:655-8. doi: 10.1016/j.steroids.2012.02.010

44. Andrisani A, Donà G, Sabbadin C, Tibaldi E, Dessole F, Bosello Travain $\mathrm{V}$, et al. Ameliorative effect of myo-inositol on red blood cell alterations in polycystic ovary syndrome: in vitro study. Gynecol Endocrinol. (2018) 34:233-7. doi: 10.1080/09513590.2017.1391207

45. Legro RS, Arslanian SA, Ehrmann DA, Hoeger KM, Murad MH, Pasquali R, et al. Diagnosis and treatment of polycystic ovary syndrome: an endocrine society clinical practice guideline. J Clin Endocrinol Metab. (2013) 98:4565-92. doi: 10.1210/jc.2013-2350 
46. Armanini D, Andrisani A, Bordin L, Sabbadin C. Spironolactone in the treatment of polycystic ovary syndrome. Expert Opin Pharmacother. (2016) 17:1713-5. doi: 10.1080/14656566.2016.1215430

47. Sabbadin C, Andrisani A, Zermiani M, Donà G, Bordin L, Ragazzi E, et al. Spironolactone and intermenstrual bleeding in polycystic ovary syndrome with normal BMI. J Endocrinol Invest. (2016) 39:1015-21. doi: 10.1007/s40618-016-0466-0

48. Armanini D, Castello R, Scaroni C, Bonanni G, Faccini G, Pellati D, et al. Treatment of polycystic ovary syndrome with spironolactone plus licorice. Eur J Obstet Gynecol Reprod Biol. (2007) 131:61-7. doi: 10.1016/j.ejogrb.2006.10.013

49. Pratesi C, Scali M, Zampollo V, Zennaro MC, De Lazzari P, Lewicka S, et al. Effects of licorice on urinary metabolites of cortisol and cortisone. J Hypertens Suppl. (1991) 9:S274-5. doi: 10.1097/00004872-19911200600121

50. Armanini D, Lewicka S, Pratesi C, Scali M, Zennaro MC, Zovato S, et al. Further studies on the mechanism of the mineralocorticoid action of licorice in humans. J Endocrinol Invest. (1996) 19:624-9. doi: 10.1007/BF033 49029

51. Armanini D, Mattarello MJ, Fiore C, Bonanni G, Scaroni C, Sartorato P, et al. Licorice reduces serum testosterone in healthy women. Steroids. (2004) 69:763-6. doi: 10.1016/j.steroids.2004.09.005

52. Ishikawa H, Ishi K, Serna VA, Kakazu R, Bulun SE, Kurita T. Progesterone is essential for maintenance and growth of uterine leiomyoma. Endocrinology. (2010) 151:2433-42. doi: 10.1210/en.2009-1225

53. Singh SS, Belland L, Leyland N, von Riedemann S, Murji A. The past, present, and future of selective progesterone receptor modulators in the management of uterine fibroids. Am J Obstet Gynecol. (2018) 218:563-72.e1. doi: 10.1016/j.ajog.2017.12.206

54. Yun AJ, Daniel SM. Sympathetic and T helper (Th)2 bias may ameliorate uterine fibroids, independent of sex steroids. Med Hypotheses. (2005) 65:11725. doi: 10.1016/j.mehy.2005.03.015

55. Isobe A, Takeda T, Wakabayashi A, Tsuiji K, Li B, Sakata M, et al. Aldosterone stimulates the proliferation of uterine leiomyoma cells. Gynecol Endocrinol. (2010) 26:372-7. doi: 10.3109/09513590903511521

56. Haan YC, Diemer FS, Van Der Woude L, Van Montfrans GA, Oehlers GP, Brewster LM. The risk of hypertension and cardiovascular disease in women with uterine fibroids. J Clin Hypertens. (2018) 20:718-26. doi: $10.1111 /$ jch. 13253

57. Laughlin-Tommaso SK, Khan Z, Weaver AL, Schleck CD, Rocca WA, Stewart EA. Cardiovascular risk factors and diseases in women undergoing hysterectomy with ovarian conservation. Menopause. (2016) 23:121-8. doi: 10.1097/GME.0000000000000506

58. Armanini D, Sabbadin C, Donà G, Bordin L, Marin L, Andrisani A, et al. Uterine fibroids and risk of hypertension: implication of inflammation and a possible role of the renin-angiotensin-aldosterone system. J Clin Hypertens. (2018) 20:727-9. doi: 10.1111/jch.13262

59. Andrisani A, Donà G, Brunati AM, Clari G, Armanini D, Ragazzi E, et al. Increased oxidation-related glutathionylation and carbonic anhydrase activity in endometriosis. Reprod Biomed Online. (2014) 28:773-9. doi: 10.1016/j.rbmo.2014.01.016

60. Hughes CL, Foster WG, Agarwal SK. The impact of endometriosis across the lifespan of women: foreseeable research and therapeutic prospects. Biomed Res Int. (2015) 2015:158490. doi: 10.1155/2015/158490

61. $\mathrm{Mu} \mathrm{F}$, Rich-Edwards J, Rimm EB, Spiegelman D, Forman JP, Missmer SA. Association Between Endometriosis and Hypercholesterolemia or Hypertension. Hypertension. (2017) 70:59-65. doi: 10.1161/HYPERTENSIONAHA.117.09056

62. Ghazi N, Arjmand M, Akbari Z, Mellati AO, Saheb-Kashaf H, Zamani Z. ${ }^{1} \mathrm{H}$ NMR-based metabolomics approaches as non- invasive tools for diagnosis of endometriosis. Int J Reprod Biomed. (2016) 14:1-8. doi: 10.29252/ijrm.14.1.1

63. Brown NJ. Aldosterone and vascular inflammation. Hypertension. (2008) 51:161-7. doi: 10.1161/HYPERTENSIONAHA.107.095489

64. Zhang L, Hao JB, Ren LS, Ding JL, Hao LR. The aldosterone receptor antagonist spironolactone prevents peritoneal inflammation and fibrosis. $L a b$ Invest. (2014) 94:839-50. doi: 10.1038/labinvest.2014.69

Conflict of Interest Statement: The authors declare that the research was conducted in the absence of any commercial or financial relationships that could be construed as a potential conflict of interest.

The reviewer FF declared a shared affiliation, with no collaboration, with several of the authors, AA, GA, LB, and GD, to the handling editor at the time of the review.

Copyright (C) 2019 Sabbadin, Andrisani, Ambrosini, Bordin, Donà, Manso, Ceccato, Scaroni and Armanini. This is an open-access article distributed under the terms of the Creative Commons Attribution License (CC BY). The use, distribution or reproduction in other forums is permitted, provided the original author(s) and the copyright owner(s) are credited and that the original publication in this journal is cited, in accordance with accepted academic practice. No use, distribution or reproduction is permitted which does not comply with these terms. 